\title{
BMJ Open Relationship between right-to-left shunt and migraine in patients with epilepsy: a single-centre, cross-sectional study in China
}

\author{
Lin Zhang, ${ }^{1}$ Xi Zhu, ${ }^{1}$ Xiangmiao Qiu, ${ }^{1}$ Yajiao Li, ${ }^{2}$ Yucheng Chen, ${ }^{2}$ Hui Wang, ${ }^{2}$ \\ Shixu He, ${ }^{1}$ Wanlin Lai, ${ }^{1}$ Anjiao Peng, ${ }^{1}$ Mingming Ning, ${ }^{3}$ Lei Chen ${ }^{1}$
}

To cite: Zhang L, Zhu X, Qiu X, et al. Relationship between right-to-left shunt and migraine in patients with epilepsy: a single-centre, cross-sectional study in China. BMJ Open 2018;8:e24144. doi:10.1136/ bmjopen-2018-024144

- Prepublication history and additional material for this paper are available online. To view these files, please visit the journal online (http://dx.doi. org/10.1136/bmjopen-2018024144).

Received 12 May 2018 Revised 28 July 2018 Accepted 27 August 2018

Check for updates

(C) Author(s) (or their employer(s)) 2018. Re-use permitted under CC BY-NC. No commercial re-use. See rights and permissions. Published by BMJ.

${ }^{1}$ Department of Neurology, West China Hospital, Sichuan University, Chengdu, Sichuan, China

${ }^{2}$ Department of Cardiology, West China Hospital, Sichuan University, Chengdu, Sichuan,

China

${ }^{3}$ Department of Neurology,

Cardio-Neurology Clinic,

Massachusetts General Hospital, Harvard Medical School, Boston, Massachusetts, USA

Correspondence to

Dr Lei Chen; leilei_25@126.com

\section{ABSTRACT}

Objectives To investigate the relationship between rightto-left shunt and migraine to account for the unexplained high prevalence of migraine in patients with epilepsy. Design This is a cross-sectional study. The diagnosis and interview process of patients with migraine was based on the International Classification of Headache Disorders-3 beta in patients with epilepsy. Participants underwent transthoracic echocardiography (TTE) with contrast medium to identify right-to-left shunt. The highest number of microbubbles were recorded in the left atrium before the complete microbubble outflow of the right atrium. A moderate-to-large shunt was defined as the presence of 10 or more microbubbles.

Setting A single-centre, cross-sectional study in China, 2015-2017.

Participants Patients with epilepsy.

Primary and secondary outcome measures The primary outcome measures were the prevalence of migraine, the prevalence of right-to-left shunt in patients with migraine and those without migraine, and the prevalence of migraine in different degrees of shunting. Results Three hundred thirty-nine participants with epilepsy who completed TTE were included in the analysis. The overall prevalence of migraine was $23.0 \%$. One-third of the migraineurs had mild right-to-left shunt and onefifth of the migraineurs had moderate-to-large right-to-left shunt. Patients with mild shunt did not have a higher prevalence of migraine than those without shunt $(26.3 \%$ vs $18.1 \%, p=0.102$ ); however, a higher prevalence of migraine was found in patients with moderate-to-large shunt $(39.0 \%$ vs $18.1 \%, 0 R=2.90,95 \% \mathrm{Cl}=1.41$ to 5.98 , $\mathrm{p}=0.003)$. Patients with migraine and patients without migraine had similar prevalence of mild shunt; however, patients with migraine had more moderate-to-large shunt (20.5\% vs $9.6 \%, p=0.002$ ). Right-to-left shunt and female were factors predicting migraine prevalence.

Conclusions One-fifth of migraineurs were correlated with moderate-to-large right-to-left shunt which could be an underlying cause of migraine in epilepsy.

\section{INTRODUCTION}

The comorbidity of migraine is common in patients with epilepsy. The lifetime occurrence of migraine was as high as $33.6 \%$ in
Strengths and limitations of this study

- Cohort study with data from a large number of patients with epilepsy.

- We first studied the relationship between the degree of shunt and migraine in patients with epilepsy.

- Transthoracic echocardiography with contrast medium was used as a tool to detect right-to-left shunt but not transoesophageal echocardiography.

patients with epilepsy, representing an overall $52 \%$ increase relative to people without epilepsy. ${ }^{12}$ Comorbid migraine may have an unfavourable effect on the prognosis of epilepsy in children and adults. ${ }^{34}$ Evidence of genetics and animal experiments have suggested similar pathophysiological mechanisms between migraine and familial epilepsy, such as genetic variants and abnormal neuronal excitability. ${ }^{5-9}$ Despite these findings, the mechanisms underlying migraine in patients with epilepsy are still controversial.

In the past two decades, right-to-left shunt, usually due to patent foramen ovale (PFO), and pulmonary arteriovenous shunt have been found to be associated with migraine. A threefold higher migraine with aura was observed in patients with right-to-left shunt compared with people without right-to-left shunt. ${ }^{10}$ There are clinical trials showing that treatment for right-to-left shunt can significantly reduce headache days although the responder rate defined as a $50 \%$ reduction in migraine attacks is not different from baseline. ${ }^{11-14}$ In addition, the meta-analyses showed that a complete resolution of migraine after treatment for right-to-left shunt occurred in $54 \%$ of migraine-with-aura cases and in 39\% of migraine-without-aura cases. ${ }^{15} 16$ Therefore, right-to-left shunt may be a fundamental cause of migraine. One of the possible mechanisms between the two 


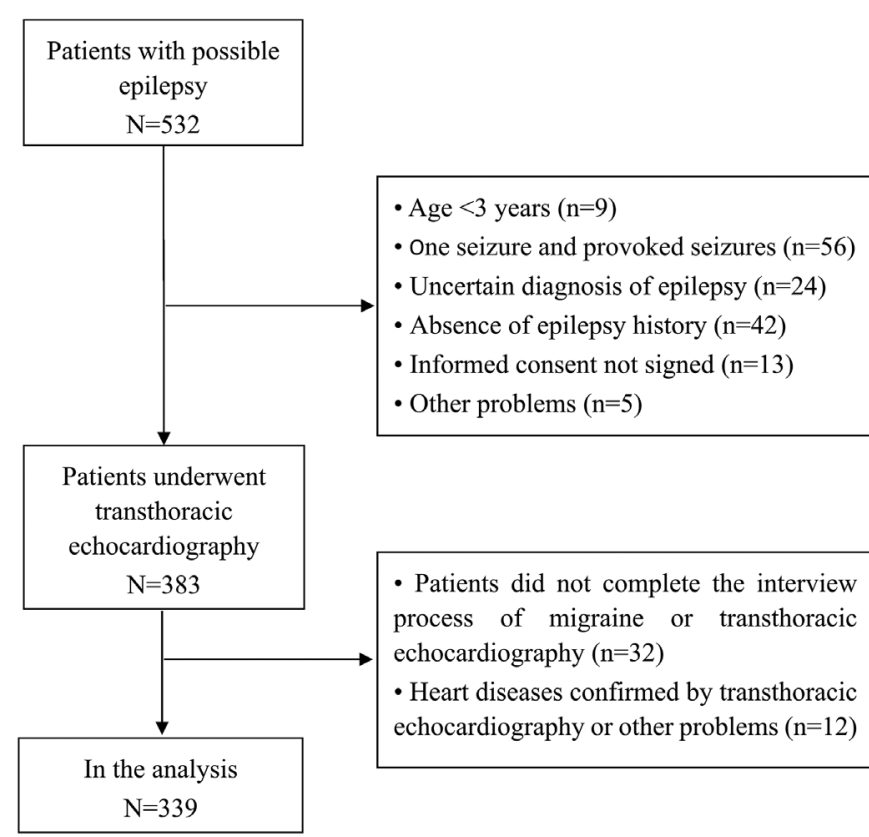

Figure 1 The flow diagram.

conditions is that vasoactive chemicals triggering migraine bypass the pulmonary filter. ${ }^{5}$ Right-to-left shunt may carry microemboli into the carotid circulation. Animal experiments showed that microemboli can trigger the aura phenomenon by inducing cortical spreading depression (CSD) ${ }^{7}$

Similarly, right-to-left shunt may also induce migraine by vasoactive chemicals and microemboli in patients with epilepsy. Therefore, our main aim was to investigate the relationship between right-to-left shunt and migraine to account for the unexplained high prevalence of migraine in epilepsy. We also investigated other migraine predictive factors, for example, sex, age, familial epilepsy, duration of epilepsy and antiepileptic drugs (AEDs).

\section{METHODS}

\section{Study design and patients}

This is a cross-sectional study. Study populations were consecutively recruited between June 2015 and December 2017 from the Department of Neurology, West China Hospital, Sichuan University, China. The inclusion criteria were as follows: (1) patients with epilepsy or possible epilepsy (patients with possible epilepsy will be followed to establish the diagnosis of epilepsy), and (2) the clinical history and medical records of epilepsy can be obtained. The exclusion criteria were as follows: (1) age <3 years; (2) inaccurate communication because of language problems; (3) patients with one seizure (because high recurrence risk factors are controversial) and provoked seizures; (4) the diagnosis of epilepsy was still undetermined when we performed the data analysis; (5) the clinical history of epilepsy including onset age of seizure, AEDs, familial history of epilepsy, seizure frequency in the last year and types of seizure could not be obtained; (6) history of heart disease except right-to-left shunt; (7) right-to-left shunt confirmed by transthoracic echocardiography (TTE) at another hospital (because there may be differences between sonographers) or transthoracic echocardiography (TTE) was unfinished; (8) informed consent not signed and (9) patients did not complete the interview process for the diagnosis of migraine.

\section{Definitions and collection of data}

A definitive diagnosis and classification of epilepsy met the criteria proposed by the International League Against Epilepsy based on the clinical history, seizure semiology, electroencephalography (EEG) and MRI findings. ${ }^{17}$ For each patient, age, symptoms of seizure, onset age of seizure, AEDs, education status, seizure onset with visual symptoms, history of febrile seizure, seizure frequency in the last year, familial history of epilepsy and types of seizure (focal onset, focal to bilateral tonic-clonic seizures or generalised onset) were recorded.

The diagnosis and interview process for patients with migraine were based on the International Classification of Headache Disorders (ICHD-3 beta). ${ }^{18}$ The number of migraine attacks, estimated duration of migraine, location, quality, intensity (Visual Analogue Scale, 0-10) and aura were recorded. Every patient was diagnosed by two neurologists separately. If the diagnosis of epilepsy and migraine was inconsistent, a third senior neurologist was asked to discuss the case further.

For children, their parents (or the children themselves if they were old enough) were asked to give specific information about epilepsy and migraine. Medical records and seizure diaries during the last 12 months were reviewed to obtain information regarding the history of epilepsy and migraine, EEG and MRI.

\section{Transthoracic echocardiography}

Participants underwent TTE with contrast medium and 1-5 MHz or 3-8 MHz multiplane transducers in Philips IE33 to identify right-to-left shunt; this was performed by two experienced sonographers who also analysed the videotapes together. Patients with other cardiac diseases (eg, valvular heart disease and cardiomyopathy) found by TTE were excluded. A microbubble bolus of agitated solution of $9 \mathrm{~mL}$ saline and $1 \mathrm{~mL}$ air was injected into one side of the patients' antecubital veins for increased sensitivity. ${ }^{19}{ }^{20}$ Patients were assessed for right-to-left shunt at rest and during the Valsalva manoeuvre and coughing. The highest number of microbubbles were recorded in the left atrium before the complete microbubble outflow of the right atrium. If three or more microbubbles appeared, a right-to-left shunt was diagnosed. The degree of shunting was defined as grade 0 if $0-2$ microbubbles occurred, grade 1 (mild) if 3-9 microbubbles occurred, grade 2 (moderate) if 10-30 microbubbles occurred and grade 3 (large) if more than 30 microbubbles occurred. ${ }^{21}$

\section{Data analysis}

Data were collected using the same standard forms in Microsoft Excel V.2013. Continuous variables were 
Table 1 Migraine prevalence in different groups

\begin{tabular}{|c|c|c|c|}
\hline Characteristics & Sample & Migraine (\%) & $P$ values \\
\hline Gender & & & 0.022 \\
\hline Male & 169 & $29(17.2)$ & \\
\hline Female & 170 & $49(28.8)$ & \\
\hline Current age, years & & & 0.039 \\
\hline$\leq 16$ & 82 & $12(11.0)$ & \\
\hline$>16$ & 257 & $66(25.7)$ & \\
\hline $\begin{array}{l}\text { Duration of epilepsy, } \\
\text { years }\end{array}$ & & & 0.001 \\
\hline$\leq 10$ & 235 & $42(17.9)$ & \\
\hline$>10$ & 104 & $36(34.6)$ & \\
\hline Onset age, years & & & 0.421 \\
\hline$\leq 16$ & 183 & $39(21.3)$ & \\
\hline$>16$ & 156 & $39(25.0)$ & \\
\hline $\begin{array}{l}\text { Seizure frequency in the } \\
\text { last year }\end{array}$ & & & 0.256 \\
\hline $0-12$ & 246 & $56(22.8)$ & \\
\hline $13-52$ & 33 & $11(33.3)$ & \\
\hline$>52$ & 28 & $7(25.0)$ & \\
\hline Unknown & 32 & $4(12.5)$ & \\
\hline Number of AEDs & & & 0.052 \\
\hline$\leq 1$ & 189 & 36 (19.0) & \\
\hline$\geq 2$ & 150 & $42(28.0)$ & \\
\hline AEDs & & & 0.299 \\
\hline
\end{tabular}

\begin{tabular}{|c|c|c|c|}
\hline Topiramate & 66 & $20(30.3)$ & \\
\hline Valproate & 126 & $29(23.0)$ & \\
\hline Levetiracetam & 94 & $26(27.7)$ & \\
\hline Oxcarbazepine & 94 & 19 (20.2) & \\
\hline Lamotrigine & 27 & $2(7.4)$ & \\
\hline Carbamazepine & 67 & $19(28.4)$ & \\
\hline Phenytoin & 20 & $4(20.0)$ & \\
\hline Phenobarbital & 12 & $2(16.7)$ & \\
\hline Seizure types & & & 0.319 \\
\hline Focal onset seizure & 68 & $10(14.7)$ & \\
\hline $\begin{array}{l}\text { Generalised onset } \\
\text { seizure and focal to } \\
\text { bilateral tonic-clonic } \\
\text { seizures }\end{array}$ & 227 & $58(25.6)$ & \\
\hline Undetermined & 44 & $10(22.7)$ & \\
\hline Familial epilepsy & 11 & $5(45.5)$ & 0.135 \\
\hline First-degree relatives & 9 & $4(44.4)$ & \\
\hline $\begin{array}{l}\text { Second-degree } \\
\text { relatives }\end{array}$ & 2 & $1(50.0)$ & \\
\hline No familial epilepsy & 328 & 73 (22.3) & \\
\hline
\end{tabular}

Right-to-left shunt

\begin{tabular}{llll} 
Grade 1 & 99 & $26(26.3)$ & 0.102 \\
Grade 2-3 & 41 & $16(39.0)$ & 0.003 \\
\hline
\end{tabular}

\begin{tabular}{|clll|}
\hline Table 1 Continued & & & \\
\hline Characteristics & Sample & Migraine (\%) & P values \\
\hline Grade 1-3 & 140 & $42(29.6)$ & 0.010 \\
Grade 0 & 199 & $36(18.1)$ & \\
\hline
\end{tabular}

AEDs, antiepileptic drugs.

compared by analysis of variance. Categorical variables were compared using the $\chi^{2}$ test or Fisher's exact test, as appropriate. Bonferroni correction was used if necessary. Logistic regression analyses were used to determine whether some variables were migraine-predicting factors. For all statistical tests, $\mathrm{p}<0.05$ was determined to be statistically significant. Statistics were performed using the SPSS statistical package V.21.0.

\section{Patient and public involvement}

The development of the research question and outcome measures were informed by published literature on PFO and migraine. Patients' priorities, experience and preferences were not involved in designing the study. Patients who agreed to enrol to the study received TTE. Two authors (LZ and XZ) disseminated the results to the study participants by interviews.

\section{RESULTS}

We carefully interviewed 532 patients with seizure(s) or (and) their parents. One hundred and sixty-one patients were excluded. The reasons for exclusion are shown in figure 1 . Three hundred and thirty-nine participants with epilepsy who completed the interview process of migraine and TTE were included in the analysis. The details of migraine features according to the ICHD- 3 beta criteria are shown in online supplementary file 1 . The overall prevalence of migraine was $23.0 \%$ (table 1 ). A greater percentage of females had migraines than males $(28.8 \%$ vs $17.2 \%, p=0.022)$. Patients $>16$ years of age seemed to have a higher prevalence of migraine than those $\leq 16$ years of age $(25.7 \%$ vs $11.0 \%, p=0.039)$. If the duration of epilepsy was more than 10 years, patients were more likely to have migraines $(34.6 \%$ vs $17.9 \%, \mathrm{p}=0.001)$. Patients with polytherapy might have more migraines $(28.0 \%$ vs $19.0 \%, \mathrm{p}=0.052)$. Patients with first-degree and second-degree relatives with epilepsy, as well as a history of febrile seizures, might not have more migraines. Patients who had visual symptoms at the onset of seizures did not have more migraines than those without visual symptoms (data not shown).

One-third of migraineurs had mild right-to-left shunt (tables 1 and 2). Patients with mild right-to-left shunt did not have a higher prevalence of migraine than those without right-to-left shunt (26.3\% vs $18.1 \%, \mathrm{OR}=1.61$, $95 \% \mathrm{CI}=0.91$ to $2.87, \mathrm{p}=0.102$ ). In our cohort, $33.3 \%$ (26 of 78) of patients with epilepsy and with migraine and $28.0 \%$ (73 of 261) of patients with epilepsy and without migraine had mild right-to-left shunt $(\mathrm{p}=0.361)$. 
Table 2 High prevalence of migraine in patients with moderate to large right-to-left shunt

\begin{tabular}{|c|c|c|c|c|}
\hline \multirow[b]{2}{*}{ Characteristics } & \multicolumn{3}{|l|}{ Right-to-left shunt } & \multirow[b]{2}{*}{$P$ values } \\
\hline & Grade 2-3 $(n=41)$ & Grade 1 (n=99) & Grade 0 (n=199) & \\
\hline Male, $n(\%)$ & $17(41.5)$ & $52(52.5)$ & $100(50.3)$ & 0.413 \\
\hline Age, years & $26.9 \pm 11.7$ & $27.6 \pm 13.7$ & $26.4 \pm 13.2$ & 0.769 \\
\hline Onset age, years & $19.3 \pm 11.2$ & $17.9 \pm 12.4$ & $17.8 \pm 12.3$ & 0.779 \\
\hline Duration, years & $8.1 \pm 8.9$ & $9.7 \pm 9.6$ & $8.7 \pm 9.1$ & 0.580 \\
\hline Familial epilepsy, n & 2 & 2 & 7 & 0.648 \\
\hline Education, n (\%) & & & & 0.772 \\
\hline $0-6$ years & $13(31.7)$ & $24(24.2)$ & $52(26.1)$ & \\
\hline $7-12$ years & $20(48.8)$ & $47(47.5)$ & $104(52.3)$ & \\
\hline$\geq 13$ years & $8(19.5)$ & 28 (28.3) & $43(21.6)$ & \\
\hline Seizure types, n (\%) & & & & 0.085 \\
\hline Focal onset & $7(17.1)$ & $22(22.2)$ & 39 (19.6) & \\
\hline $\begin{array}{l}\text { Generalised onset seizure } \\
\text { and focal to bilateral tonic- } \\
\text { clonic seizures }\end{array}$ & $32(78.0)$ & $69(69.7)$ & $126(63.3)$ & \\
\hline Undetermined & $2(4.9)$ & $8(8.1)$ & $34(17.1)$ & \\
\hline $\begin{array}{l}\text { Seizure frequency in the last } \\
\text { year, } n(\%)\end{array}$ & & & & 0.284 \\
\hline $0-12$ & 27 (65.9) & $71(71.7)$ & $148(71.4)$ & \\
\hline $13-52$ & $3(7.3)$ & $14(14.1)$ & $16(8.0)$ & \\
\hline$>52$ & $6(14.6)$ & $8(8.1)$ & $14(7.0)$ & \\
\hline Unknown & $5(12.2)$ & $6(6.1)$ & $21(14.1)$ & \\
\hline Number of used AEDs & & & & 0.139 \\
\hline$\leq 1, \mathrm{n}(\%)$ & $25(61.0)$ & 47 (47.5) & $117(58.8)$ & \\
\hline$\geq 2, \mathrm{n}(\%)$ & $16(39.0)$ & $52(52.5)$ & $82(41.2)$ & \\
\hline AEDs & & & & 0.735 \\
\hline Topiramate & 10 & 21 & 35 & \\
\hline Valproate & 8 & 45 & 74 & \\
\hline Levetiracetam & 15 & 31 & 48 & \\
\hline Oxcarbazepine & 12 & 34 & 48 & \\
\hline Lamotrigine & 4 & 8 & 15 & \\
\hline Carbamazepine & 8 & 28 & 31 & \\
\hline Phenytoin & 1 & 6 & 13 & \\
\hline Phenobarbital & 1 & 4 & 7 & \\
\hline Migraine, n (\%) & $16(39.0)$ & $26(26.3)$ & $36(18.1)$ & $0.010 *$ \\
\hline
\end{tabular}

The continuous data are presented as mean \pm SD.

*Bonferroni correction shows statistical differences in grade 2-3 versus grade 0 .

AEDs, antiepileptic drugs.

One-fifth of migraineurs had moderate-to-large rightto-left shunt (tables 1 and 2). Patients with moderateto-large right-to-left shunt had a higher prevalence of migraine than those without right-to-left shunt $(39.0 \%$ vs $18.1 \%, \mathrm{OR}=2.90,95 \% \mathrm{CI}=1.41$ to $5.98, \mathrm{p}=0.003)$. Twenty-one per cent (16 of 78) of patients with epilepsy and with migraine had moderate-to-large right-to-left shunt; whereas only $10 \%$ (25 of 261) of patients with epilepsy and without migraine had moderate-to-large right-to-left shunt $(\mathrm{OR}=2.44,95 \% \mathrm{CI}=1.23$ to $4.84, \mathrm{p}=0.002)$. After controlling for potential confounders, there were significant differences of migraine prevalence between the groups with right-to-left shunt grade 2-3 versus the group with right-to-left shunt grade 0 (table 2). Therefore, moderate-to-large right-to-left shunt but not mild rightto-left shunt was associated with migraine in patients with epilepsy.

We used a logistic regression analysis to identify the possible factors predicting migraine prevalence, such as gender, duration of epilepsy, current age, right-to-left 
Table 3 Logistic regression analysis for migraine predictors

\begin{tabular}{|c|c|c|c|c|}
\hline \multirow[b]{2}{*}{ Characteristics } & \multirow[b]{2}{*}{ OR } & \multicolumn{2}{|c|}{$95 \% \mathrm{Cl}$} & \multirow[b]{2}{*}{$P$ values } \\
\hline & & Lower & Upper & \\
\hline Male & 0.518 & 0.302 & 0.891 & 0.017 \\
\hline Current age, years & 0.978 & 0.956 & 1.001 & 0.057 \\
\hline Right-to-left shunt & 2.200 & 1.274 & 3.797 & 0.005 \\
\hline $\begin{array}{l}\text { Duration of epilepsy, } \\
\text { years }\end{array}$ & 0.974 & 0.945 & 1.004 & 0.085 \\
\hline Familial epilepsy & 2.828 & 0.765 & 10.453 & 0.119 \\
\hline $\begin{array}{l}\text { Number of } \\
\text { antiepileptic drugs }>1\end{array}$ & 1.354 & 0.784 & 2.340 & 0.277 \\
\hline
\end{tabular}

shunt and number of AEDs (table 3). Right-to-left shunt and female were factors predicting migraine prevalence. Although patients with duration of epilepsy $>10$ years and older age had a higher migraine prevalence (table 1), the logistic regression analysis showed no significant statistical difference.

PFO closure were performed in seven patients. Four patients were followed for 6 months. We found that PFO closure fully controlled migraine in two of four cases. Before PFO closure, the two patients had more than 11 migraine attacks in the last year. More interesting, the frequency of seizures in three patients with migraine after PFO closure decreased significantly.

\section{DISCUSSION}

This is the first study to evaluate the relationship between migraine and right-to-left shunt in a large number of patients with epilepsy. Our main findings were as follows: (1) one-third of the migraineurs had mild right-to-left shunt and one-fifth of the migraineurs had moderate-tolarge right-to-left shunt; (2) patients with mild right-to-left shunt did not have a higher prevalence of migraine than those without right-to-left shunt; (3) moderate-to-large right-to-left shunt was associated with migraine in patients with epilepsy and (4) right-to-left shunt and female were factors predicting migraine prevalence in patients with epilepsy.

Migraine in patients without epilepsy has been proved to be associated with CSD and activation of the trigeminovascular system and its constituent neuropeptides. ${ }^{22}$ In addition, recent studies also suggested the cortical excitatory/inhibitory imbalance due to neuronal and glial ion channels renders patients more vulnerable to an headache attack. ${ }^{22}$ Migraine in patients with epilepsy is not only associated with the aforementioned mechanisms, but also is usually considered to be associated with abnormal neuronal excitability induced by genetic variants. ${ }^{5-9}$ However, the high prevalence of migraine in patients with epilepsy cannot be fully explained by genetic variants. In the past two decades, right-to-left shunt has been found to be associated with migraine in patients without epilepsy. Our major hypothesis was that right-to-left shunt might also be one of the pathophysiological mechanisms of migraine in patients with epilepsy. The results showed that mild right-to-left shunt was not associated with migraine in patients with epilepsy. Almost one-fifth of migraines were correlated with moderate-to-large right-to-left shunt which could be an underlying cause of migraine in epilepsy. The mechanisms of the relationship between moderate-tolarge right-to-left shunt and migraine remains speculative. A previous study showed that larger right-to-left shunt tended to trigger migraine attacks because of the increased extent of the shunt. ${ }^{23}$ Larger shunt allows harmful circulatory factors, such as vasoactive substances and microemboli which are eliminated by lung, to bypass the pulmonary filter and travel directly from the venous to the cerebral artery. Right-to-left shunt and higher rates of Doppler-detected cerebral microemboli have been reported to be associated. ${ }^{24}$ In an animal experiment, air microemboli are able to trigger CSD and migraine aura often with transient microvascular occlusion that is insufficient to cause a permanent ischaemic lesion. ${ }^{7}$ In humans, diagnostic microbubble injection also induced attacks of migraine with aura. $^{25}$ Therefore, microemboli may be a key role linking CSD, migraine and moderate-to-large shunt.

Interestingly, CSD increases neuronal excitability and triggers ictaform activity in neuronal tissues with partial impairment of inhibitory. ${ }^{9}$ CSD also triggers migraine headache by activating neuronal Panx1 channels which contributes to neuronal hyperactivity in seizures. ${ }^{2627}$ These findings may explain why migraine may have an unfavourable effect on the prognosis of

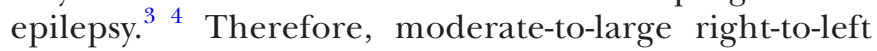
shunt may not only be an underlying cause of migraine but also an adverse prognostic factor in patients with epilepsy. Whether moderate-to-large right-to-left shunt is an adverse prognostic factors in epilepsy should also be investigated.

The effect of PFO closure for migraine treatment still remains controversial. Randomised controlled trials (RCTs) looking at the effect of PFO closure on migraine were initially conducted. There are clinical trials showing that treatment for right-to-left shunt has not increased responder rate defined as 50\% reduction in migraine attacks; however, it has significantly reduced headache days. ${ }^{11-14}$ Similarly to the stroke trials, they had initial negative RCTs. With better patient selection and longer follow-up, subsequent trials may show more cessation of migraine headache. ${ }^{28}$ In our cohort, we found that PFO closure fully controlled migraine in two of four cases. More interestingly, the frequency of seizures in three patients with migraine after surgery decreased significantly. Although these cases need to be explained with caution, it is worth investigating in the future whether PFO closure improves migraine attacks and seizure outcomes. In addition, given the subjective nature of migraines and their vulnerability to bias and the placebo effect, perhaps a large, well-designed sham controlled trial with modern 
devices is required. If treatment for right-to-left shunt has been shown to be definitely effective, right-to-left shunt should be screened in patients with epilepsy and with migraine.

This study has some limitations. First, transoesophageal echocardiography (TEE) is a gold standard diagnostic tool with a sensitivity and specificity of $80 \%-100 \%$ for the detection of a right-to-left shunt. ${ }^{19} 20$ The contrast TTE used by us has a lower sensitivity than TEE. ${ }^{19}$ However, harmonic imaging improves sensitivity for the detection of right-to-left shunt, ranging from 66\% to 100\%. ${ }^{19} 202930$ In addition, TTE is not inferior to TEE in the assessment of large shunt because TEE may miss large shunt detected by TTE. ${ }^{30}$ In our study, moderate-to-large right-to-left shunt was more likely to be associated with migraine; therefore, TTE might not be an inferior choice to TEE. Second, the population might be biased because a few patients did not complete TTE. However, there were no differences in demographic characteristics between patients who did or did not refuse TTE and the conclusions of our study should not be affected. Third, only Chinese people were recruited, and this would limit the generalisability of results.

\section{CONCLUSIONS}

Moderate-to-large right-to-left shunt but not mild rightto-left shunt was associated with migraine. On the basis of these results, patients with epilepsy and with migraine should be screened for a right-to-left shunt if treatment for a right-to-left shunt has been shown to be effective in migraine. Whether moderate-to-large right-to-left shunt is an adverse prognostic factor in epilepsy should also be investigated.

\section{Acknowledgements We thank Dr Qi Si for her help.}

Contributors LC and MN conceived and designed the work. LZ and XZ were involved in data collection, data analysis and interpretation. LZ drafted the manuscript. $\mathrm{LC}$ involved in critical revision of the article and final approval of the version to be published. XZ, XQ, SH, AP, WL, YL, YC and HW were involved in the data collection and final approval of the version to be published. All authors have agreed to be accountable for all aspects of the work.

Funding This study were provided by Technology and Science Department of Sichuan Province (No.2017HH0103) and China Association Against Epilepsy (No.2016005).

Competing interests None declared.

Patient consent Obtained.

Ethics approval The protocol was approved by the Ethics Committee of the West China Hospital of Sichuan University.

Provenance and peer review Not commissioned; externally peer reviewed. Data sharing statement № additional data available.

Open access This is an open access article distributed in accordance with the Creative Commons Attribution Non Commercial (CC BY-NC 4.0) license, which permits others to distribute, remix, adapt, build upon this work non-commercially, and license their derivative works on different terms, provided the original work is properly cited, appropriate credit is given, any changes made indicated, and the use is non-commercial. See: http:// creativecommons.org/licenses/by-nc/4.0/.

\section{REFERENCES}

1. Keezer MR, Bauer PR, Ferrari MD, et al. The comorbid relationship between migraine and epilepsy: a systematic review and metaanalysis. Eur J Neurol 2015;22:1038-47.

2. Le H, Tfelt-Hansen P, Russell MB, et al. Co-morbidity of migraine with somatic disease in a large population-based study. Cephalalgia 2011;31:43-64

3. Dragoumi P, Tzetzi O, Vargiami E, et al. Clinical course and seizure outcome of idiopathic childhood epilepsy: determinants of early and long-term prognosis. BMC Neurol 2013;13:206.

4. Velioğlu SK, Boz C, Ozmenoğlu M. The impact of migraine on epilepsy: a prospective prognosis study. Cephalalgia 2005;25:528-35.

5. Finocchi C, Del Sette M. Migraine with aura and patent foramen ovale: myth or reality? Neurol Sci 2015;36 Suppl 1(Suppl 1):61-6.

6. Ludvigsson P, Hesdorffer D, Olafsson E, et al. Migraine with aura is a risk factor for unprovoked seizures in children. Ann Neurol 2006;59:210-3.

7. Nozari A, Dilekoz E, Sukhotinsky I, et al. Microemboli may link spreading depression, migraine aura, and patent foramen ovale. Ann Neurol 2010;67:221-9.

8. Winawer MR, Connors R. EPGP Investigators. Evidence for a shared genetic susceptibility to migraine and epilepsy. Epilepsia 2013;54:288-95.

9. Eickhoff M, Kovac S, Shahabi P, et al. Spreading depression triggers ictaform activity in partially disinhibited neuronal tissues. Exp Neurol 2014;253:1-15.

10. Wammes-van der Heijden EA, Tijssen CC, Egberts AC. Right-to-left shunt and migraine: the strength of the relationship. Cephalalgia 2006;26:208-13.

11. Xing YQ, Guo YZ, Gao YS, et al. Effectiveness and Safety of Transcatheter Patent Foramen Ovale Closure for Migraine (EASTFORM) Trial. Sci Rep 2016;6:39081.

12. Tobis JM, Charles A, Silberstein SD, et al. Percutaneous Closure of Patent Foramen Ovale in Patients With Migraine: The PREMIUM Trial. J Am Coll Cardiol 2017;70:2766-74.

13. Dowson A, Mullen MJ, Peatfield R, et al. Migraine Intervention With STARFlex Technology (MIST) trial: a prospective, multicenter, doubleblind, sham-controlled trial to evaluate the effectiveness of patent foramen ovale closure with STARFlex septal repair implant to resolve refractory migraine headache. Circulation 2008;117:1397-404.

14. Mattle HP, Evers S, Hildick-Smith D, et al. Percutaneous closure of patent foramen ovale in migraine with aura, a randomized controlled trial. Eur Heart J 2016;37:2029-36.

15. Kanwar SM, Noheria A, DeSimone CV, et al. Coincidental impact of transcatheter patent foramen ovale closure on migraine with and without aura - a comprehensive meta-analysis. Clin Trials Regul Sci Cardiol 2016;15:7-13.

16. Shi YJ, Lv J, Han XT, et al. Migraine and percutaneous patent foramen ovale closure: a systematic review and meta-analysis. BMC Cardiovasc Disord 2017;17:203.

17. Fisher RS, Cross JH, French JA, et al. Operational classification of seizure types by the International League Against Epilepsy: Position Paper of the ILAE Commission for Classification and Terminology. Epilepsia 2017;58:522-30.

18. Ettlin DA. Headache Classification Committee of the International Headache Society (IHS). The International Classification of Headache Disorders, 3rd edition (beta version). Cephalalgia 2013;33:629-808.

19. Cotter PE, Martin PJ, Belham M. Improved sensitivity of transthoracic contrast echocardiography in the detection of right-to-left shunts. $J$ Am Soc Echocardiogr 2010;23:578.

20. Ha JW, Shin MS, Kang S, et al. Enhanced detection of rightto-left shunt through patent foramen ovale by transthoracic contrast echocardiography using harmonic imaging. Am J Cardiol 2001;87:669-71.

21. Mas JL, Arquizan C, Lamy C, et al. Recurrent cerebrovascular events associated with patent foramen ovale, atrial septal aneurysm, or both. N Engl J Med 2001;345:1740-6.

22. Pietrobon D, Moskowitz MA. Pathophysiology of migraine. Annu Rev Physiol 2013;75:365-91.

23. Tembl J, Lago A, Sevilla T, et al. Migraine, patent foramen ovale and migraine triggers. J Headache Pain 2007;8:7-12.

24. Rodriguez RA, Sinclair B, Weatherdon D, et al. Patent foramen ovale and brain microembolization during scoliosis surgery in adolescents. Spine 2001;26:1719-21.

25. Dinia L, Roccatagliata L, Bonzano L, et al. Diffusion MRI during migraine with aura attack associated with diagnostic microbubbles injection in subjects with large PFO. Headache 2007;47:1455-6.

26. Karatas H, Erdener SE, Gursoy-Ozdemir Y, et al. Spreading depression triggers headache by activating neuronal Panx1 channels. Science 2013;339:1092-5. 
27. Santiago MF, Veliskova J, Patel NK, et al. Targeting pannexin1 improves seizure outcome. PLoS One 2011;6:e25178.

28. Mojadidi MK, Zaman MO, Elgendy IY, et al. Cryptogenic Stroke and Patent Foramen Ovale. J Am Coll Cardiol 2018;71:1035-43.

29. Clarke NR, Timperley J, Kelion AD, et al. Transthoracic echocardiography using second harmonic imaging with Valsalva manoeuvre for the detection of right to left shunts. Eur $J$ Echocardiogr 2004;5:176-81.

30. Daniëls C, Weytjens C, Cosyns B, et al. Second harmonic transthoracic echocardiography: the new reference screening method for the detection of patent foramen ovale. Eur $J$ Echocardiogr 2004;5:449-52. 